\title{
Highly Sensitive Twist Sensor Based on Partially Silver Coated Hollow Core Fiber Structure
}

\author{
Dejun Liu \\ Technological University Dublin, dejun.liu@tudublin.ie \\ Rahul Kumar \\ Tyne, U.K.

\section{Fangfang Wei} \\ Technological University Dublin, fangfang.wei@tudublin.ie
}

Department of Mathematics, Physics and Electrical Engineering, Northumbria University, Newcastle Upon

See next page for additional authors

Follow this and additional works at: https://arrow.tudublin.ie/engschmanart

Part of the Electrical and Computer Engineering Commons

\section{Recommended Citation}

Liu, D., Kumar, R. \& Wei, F. (2018). Highly Sensitive Twist Sensor Based on Partially Silver Coated Hollow Core Fiber Structure. Journal of Lightwave Technology, vol. 36, no. 17, pg. 3672-3677. doi:10.1109/ JLT.2018.2842111

This Article is brought to you for free and open access by the School of Manufacturing and Design Engineering at ARROW@TU Dublin. It has been accepted for inclusion in Articles by an authorized administrator of ARROW@TU Dublin. For more information, please contact arrow.admin@tudublin.ie, aisling.coyne@tudublin.ie, gerard.connolly@tudublin.ie.

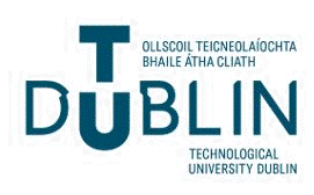




\section{Authors}

Dejun Liu, Rahul Kumar, Fangfang Wei, Wei Han, Arun Mallik, Jinhui Yuan, Chongxiu Yu, Feng Li, Zhengyong Liu, Hwa-Yaw Tam, Gerald Farrell, Yuliya Semenova, and Qiang Wu 


\title{
Highly sensitive twist sensor based on partially silver coated hollow core fiber structure
}

\author{
Dejun Liu, Rahul Kumar, Fangfang Wei, Wei Han, Arun Kumar Mallik, Jinhui Yuan, Chongxiu Yu, \\ Zhe Kang, Feng Li, Zhengyong Liu, Hwa-Yaw Tam, Gerald Farrell, Yuliya Semenova, and Qiang Wu
}

\begin{abstract}
Interferometer based on multiple beam interferences inside a hollow core fiber (HCF) structure (also known as an anti-resonant reflecting optical waveguide) has been attracting interest of many researchers due to its periodic transmission spectrum containing high quality factor spectral dips. Functionalized HCF structures have been demonstrated for a wide range of applications in humidity, magnetic field and bio-sensing. Here, we report a new application of the HCF based structure with a partial silver coating layer for sensing of twist. It is configured by fusion splicing a section of $4.5-\mathrm{mm}$ long HCF between two standard single mode fibers (SMFs), followed by a sputter-coating of a very thin layer of silver on one side of the HCF surface. It is found that the spectral response of the partially silver coated HCF structure is very sensitive to the changes of input light polarization. An increase in sensitivity of the fiber structure to twist after deposition of the silver coating when twist is applied to both the SMF and HCF sections is demonstrated by comparison with an uncoated HCF fiber structure. Experimental results show that twisting of the HCF section results in much greater changes in a selected dip's strength compared to that in the case of twisting the SMF section of the structure. The proposed HCF fiber sensors with $4.5 \mathrm{~nm}$ and $6.7 \mathrm{~nm}$-thick silver layers show the highest sensitivities of $0.647 \mathrm{~dB} /{ }^{\circ}$ and $0.717 \mathrm{~dB} /{ }^{\circ}$ in the twist angles range of up to $10^{\circ}$. To the best of our knowledge, this is the highest twist sensitivity reported for intensity
\end{abstract}

Manuscript received XXXX; revised December XXXX; accepted XXXX. Date of publication XXXX; date of current version XXXX. This work was supported by DIT FIOSRAIGH 2012 (Dean of Graduate Students Award) and Open Fund, State Key Laboratory of Advanced Optical Communication Systems and Networks, Shanghai Jiao Tong University, China; (Corresponding author: Q. Wu and J. Yuan).

D. Liu, F. Wei, W. Han, A. K. Mallik, G. Farrell, and Y. Semenova are with the Photonics Research Centre, Dublin Institute of Technology, Kevin Street, Dublin 8, Ireland (email: dejun.liu@mydit.ie; fangfang.wei@mydit.ie; d14125980@mydit.ie; d13125763@mydit.ie; yuliya.semenova@dit.ie; gerald.farrell@dit.ie).

Q. Wu and R. Kumar are with the Department of Mathematics, Physics and Electrical Engineering, Northumbria University, Newcastle Upon Tyne, NE1 8ST, United Kingdom (email: qiang.wu@northumbria.ac.uk; rahul.kumar@northumbria.ac.uk). Q. Wu is also with the School of Physics \& Optoelectronic Engineering, Nanjing University of Information Science \& Technology, Nanjing 210044, China

J. Yuan and $\mathrm{C}$. Yu are with the Beijing University of Posts and Telecommunications, Beijing 100876, China (email: yuanjinhui81@163.com; cxyu@bupt.edu.cn).

Z. Kang and F. Li are with the Photonics Research Centre, Department of Electronic and Information Engineering, The Hong Kong Polytechnic University, Hong Kong (email: zhe.kang@polyu.edu.hk; lifeng.hk@gmail.com).

Z. Liu and H. Y. Tam is with Photonics Research Centre, Department of Electrical Engineering, The Hong Kong Polytechnic University, Hong Kong (zhengyong.liu@connect.polyu.hk; hwa-yaw.tam@polyu.edu.hk). modulation based fiber sensors. Moreover, the proposed sensor offers excellent measurement repeatability.

Index Terms-Optical fiber sensors, optical interferometry, optical fiber applications, optical polarization, silver.

\section{INTRODUCTION}

Structural health monitoring and shape sensing have been

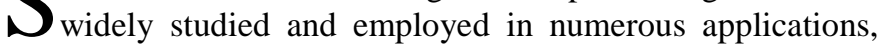
including monitoring of bridges, buildings, tunnels, dams and pipelines, for the purpose of warning about the impending abnormal conditions or accidents at an early stage to avoid casualties and to provide maintenance and repair advice [1-2]. Twist/torsion is one of the key parameters that frequently needs to be considered in structural health monitoring. Compared with traditional electromagnetic and electronic sensors, optical fiber based twist/ torsion sensors have attracted significant attention due to their unique advantages such as miniature size, immunity to electromagnetic interference and remote sensing capabilities.

To date, a number of optical fiber based twist sensors have been reported. In general, those sensors can be mainly categorized into the following types, depending on the sensing fiber structure used: fiber gratings [3-8], polarization maintaining fibers (PMFs) [9-12], photonic crystal fibers (PCFs) [13-17] and customized and specially designed fiber based sensors [18-20]. However, fiber grating based twist sensors suffer from relatively low twist sensitivity, most importantly, the fabrication process for such gratings is complex and expensive since it normally requires using an Excimer laser and phase masks. PCF and PMF based twist sensors are reported with much higher twist sensitivity by combining them with Sagnac interferometers, but those sensors need relatively complex signal interrogation systems, which might limit their potential applications. The customized fiber based sensors have the disadvantages of high cost and requiring complex and expensive fabrication setups.

Hollow core fiber (HCF) has been widely reported for use in optical fiber sensing applications. For example, a Fabry-Perot interferometer (FPI) based sensor was fabricated by incorporating a micro sized air-gap in the HCF to measure a range of environmental parameters, such as temperature and thermo-optic coefficients [21-23]. Most recently, a HCF based interferometer with high quality factor transmission spectrum has been reported [24]; functionalized HCF structures have 
been demonstrated for humidity sensing, magnetic field monitoring and bio-sensing [25-27]. In this paper, we report a novel high sensitivity twist sensor based on a commercial HCF structure utilizing an interferometric approach, enhanced by the addition of a thin coating layer of silver on one side of the HCF. A maximum twist sensitivity of $0.717 \mathrm{~dB} /{ }^{\circ}$ has been experimentally demonstrated.

\section{TheOretical Model AND ANAlysis}

Fig. 1(a) illustrates a schematic diagram of the proposed SMF-HCF-SMF fiber structure with partial silver coating at the outer cladding of HCF. The light interference and transmission are illustrated only for the top half of the structure for the sake of clarity. Fig. 1(b) shows a scanning electron microscope (SEM) image of the HCF used and Fig. 1(c) shows a schematic diagram of the HCF cross-section after its partial silver coating (one sided coating). Since the refractive index (RI) of the air core (assuming air has a RI of 1 ) is smaller than that of the HCF silica cladding, the optical mode is leaky during its propagation along the HCF. The light incident from the input SMF will be reflected at the interfaces between air core/cladding and cladding/silver/outer air. Multiple reflections take place and there are thus multiple beam interferences within the air core of the HCF, until the propagating beams enter the output SMF.

(a)

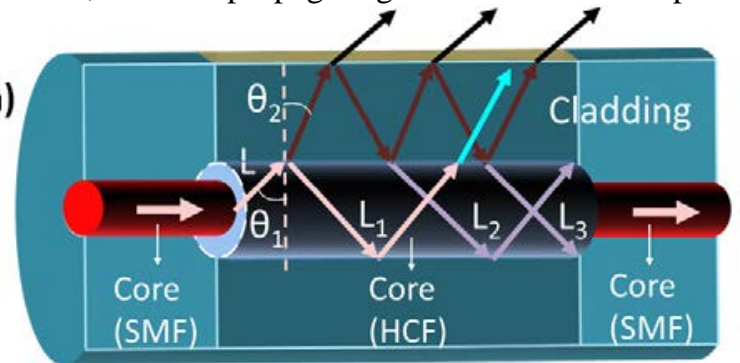

(b)

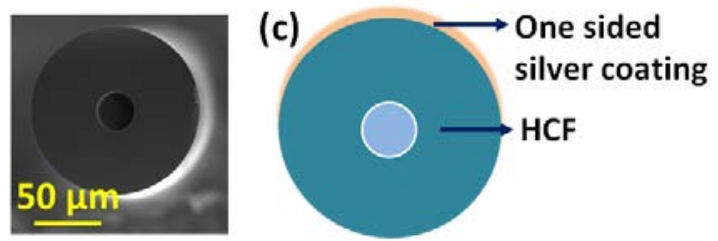

FIG. 1. (a) A schematic diagram of the proposed SMF-HCF-SMF fiber structure, showing the multiple beams interference inside the hollow core; (b) an SEM image of the used HCF at the cross-section; and (c) a schematic diagram of the HCF cross-section after coating with silver layer.

Let us assume $A$ is the amplitude of the incident light ray $(L)$, $\theta_{1}$ and $\theta_{2}$ are the incident and refracted angles at the interface between the air core and cladding. If the HCF has a cladding thickness $d$ and RI $n$, then the phase difference between the two adjacent reflected light rays inside the air core of the HCF $\left(L_{1}\right.$, $L_{2}, L_{3} \ldots$ ) can be calculated by

$\delta=\frac{4 \pi}{\lambda} n d \cos \theta_{2}$

The amplitudes of the reflected light $L_{1}, L_{2}, L_{3} \ldots L_{j} \ldots$ can be described as:

$r_{1} A, r_{2} \rho^{2} A e^{i \delta}, r_{2}\left(-r_{1} r_{2}\right) \rho^{2} A e^{i 2 \delta} \cdots, r_{2}\left(-r_{1} r_{2}\right)^{m-1} \rho^{2} A e^{i m \delta}, \cdots$,

where $r_{1}$ and $r_{2}$ are the reflection coefficients at the interface between the inner air/cladding and cladding/silver/outer air respectively, $\rho$ represents refraction coefficient at the interface between the inner air and fiber cladding, and $m$ is a positive integer. Then the final amplitude of the light propagating in the hollow core can be determined by summing all the reflected amplitudes as follows:

$$
\begin{aligned}
& \frac{A_{r}=r_{1} A+r_{2} \rho^{2} A e^{i \delta}+r_{2}\left(-r_{1} r_{2}\right) \rho^{2} A e^{i 2 \delta}}{+\cdots+r_{2}\left(-r_{1} r_{2}\right)^{m-1} \rho^{2} A e^{i m \delta}+\cdots} \\
& \quad=\frac{r_{1}+r_{2} e^{i \delta}}{1+r_{1} r_{2} e^{i \delta}} \mathrm{A}
\end{aligned}
$$

The light intensity transmitted in the hollow core is hence given as

$$
I_{r}=\left|A_{r}\right|^{2}
$$

As can be seen from the above equations, the transmitted light intensity only depends on the incident light amplitude and on the refraction and reflection coefficients at the interfaces between the air core /cladding and cladding/silver/outer air. It is noted that for the lower half of the HCF structure the transmitted light intensity could be also calculated using above equations but with a different value of $r_{2}$. Fig. 2 shows a family of simulated spectral responses of the structure for varying reflection coefficients $r_{2}$ while assuming $r_{1}$ constant. In the simulation, the diameters of the air core and cladding are $30 \mu \mathrm{m}$ and $126 \mu \mathrm{m}$ respectively. The incident angle $\theta_{1}$ is $80.8^{\circ}$. The incidence angle is chosen for a typical single mode fiber with $\mathrm{NA}=0.16$, where $r_{1}=-0.74$. The refractive indices of the air and silica cladding are 1 and 1.444 respectively. As shown in Fig. 2, as $r_{2}$ deviates from $r_{1}$ further, the dip's strength decreases significantly.

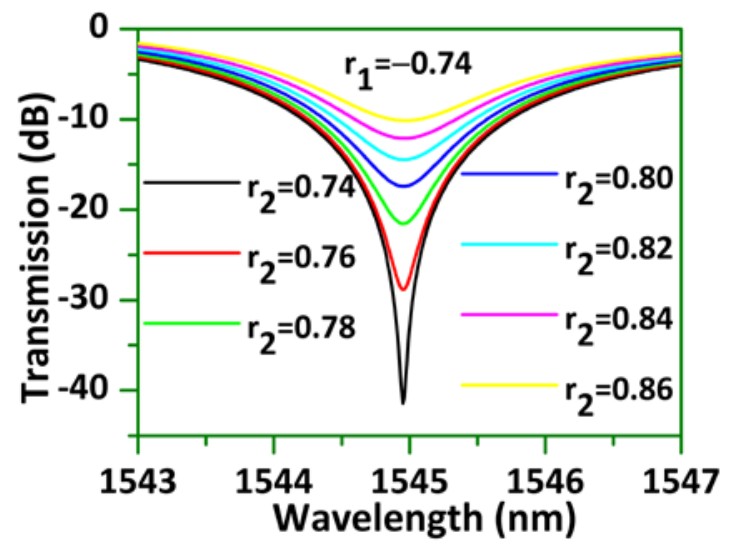

FIG. 2. Simulated spectral responses of the HCF structure with a constant reflection coefficient $r_{1}$ and varying reflection coefficient $r_{2}$.

\section{RESUlTS AND DISCUSSION}

Here we report on the results of the experimental investigation of the influence of twist on the transmission spectrum of the partially silver-coated HCF structure. Firstly, a short section of a HCF (inner air core diameter $\sim 30 \mu \mathrm{m}$, outer cladding diameter $\sim 126 \mu \mathrm{m}$ ) with a length of $4.5 \mathrm{~mm}$ was fusion spliced between two SMFs. A length of $4.5 \mathrm{~mm}$ was chosen based on the previous experience, which demonstrated that this particular HCF length resulted in a relatively high Q factor and low loss. The splicing power and the gaps between the SMFs and HCF were carefully chosen to avoid the collapse of the hollow core during fusion splicing. In our experiment, a Fujikura $70 \mathrm{~S}$ 
splicer was used, and its arc power was set to be STD-85 bit, arc time was $0.4 \mathrm{~s}$ and the arc gap was $13 \mu \mathrm{m}$. Once the fiber structure was fabricated, a thin layer of silver was coated on one side of the HCF surface using a sputter-coating machine (Quorum Technologies Q150RS). For our experiments 6 fiber structure samples were fabricated with different coating thicknesses. During the coating process, the HCF fiber was placed horizontally in the sputtering chamber and coated for a fixed period of time. Since the fiber is fixed within the chamber, only one side of the HCF is coated with silver. It is noted that the coating thickness is unevenly distributed over the surface, with the maximum thickness at the top of the cylinder's cross-section, decreasing towards both sides (Fig. 1(c)).

Because silica is not a conductor and only a very thin layer (around $10 \mathrm{~nm}$ ) of silver is coated on the HCF fiber surface, it is difficult to measure the actual thickness of the silver coating unless using a high resolution SEM. Therefore in our experiment the coating thickness is a calibrated value on a glass slide calculated as $6.7 \mathrm{~nm} /$ minute $\times$ Time as shown in Fig. 3.

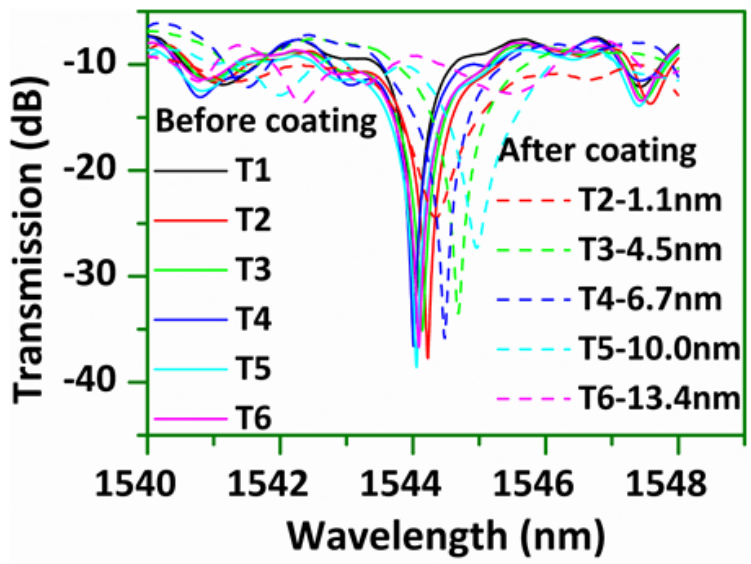

FIG. 3. Measured spectral responses of the HCF based fiber structures before and after deposition of the silver coating.

Fig. 3 illustrates the influence of the silver coating thickness on the sensor's spectral response, where the largest transmission dips for all the six samples (T1 to T6) are shown before and after application of the silver coating by adjusting the polarization state of the input light using a manual polarization controller (PC, DPC5000). As one can see from the figure, for the fiber samples without the silver coating, spectral responses have similar transmission dips with a maximum variation between the central dip wavelengths of $0.22 \mathrm{~nm}$ and dip strength variation of $4.4 \mathrm{~dB}$, which proves the HCF based fiber structure shows good reproducibility. The observed minor variations in the transmission spectra can be attributed to minor differences in each fiber fusion splice conditions, due to minor splice power variations, and by different degrees of flatness and cleanness of the fiber ends after cleaving and fiber alignment during the fiber fusion splicing process. With the increase of the coating time from 10 to $120 \mathrm{~s}$ (equivalent to a coating thickness increase from 1.1 to $13.4 \mathrm{~nm}$ ), the transmission dips gradually move toward longer wavelengths. The largest two wavelength dips after coating are observed when the coating times are $40 \mathrm{~s}$ (T3) and $60 \mathrm{~s}$ (T4), where the coating thickness is calculated to be 4.5 and $6.7 \mathrm{~nm}$, respectively.
The uncoated and partially silver-coated HCF fiber structures were then placed within the experimental setup shown in Fig. 4. In our experiment, we investigated the effect of twist separately for the SMF section only (Section 1 in the figure) and also for a section containing the HCF (Section 2). To realize this, twisting of the fiber structure was carried out using two fiber rotators with the fiber firmly held in place at two points by fiber holders, marked as $\mathrm{H} 1$ and $\mathrm{H} 2$ in Fig. 4. Rotator 1 was used for twisting the SMF section (Section 1) only and rotator 2 was used for twisting the fiber structure section including the whole length of the HCF (Section 2). Both Section 1 and Section 2 have the same length of 20 centimeters, and the twist angle resolution of the rotators is about one degree. Before the fiber was fixed onto the stages, a weight of $14.6 \mathrm{~g}$ was used between rotator 2 and a third fiber holder (H3), to keep the fiber straight and to ensure that a fixed strain has been applied to the fiber structure during the twist experiment. Light from a broadband light source (BBS) was launched into the HCF based structure and the transmitted light was measured by an optical spectrum analyzer (OSA). A PC (DPC5500) was used to achieve linear polarized light and to adjust the polarization state of the light before it enters the twisted fiber sections.

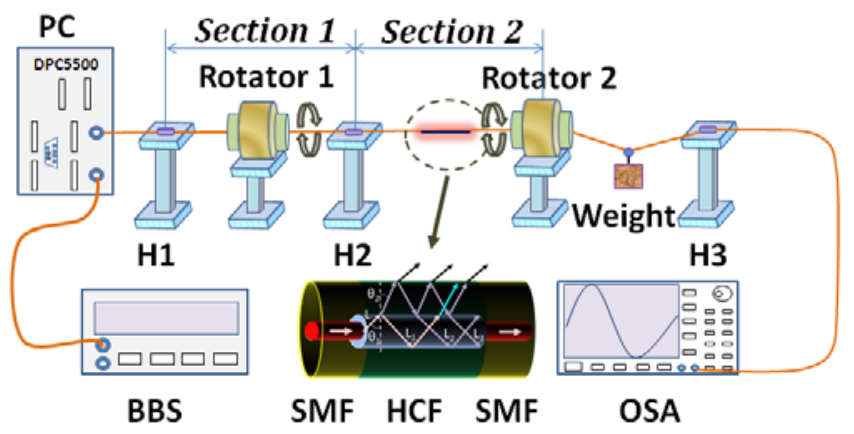

FIG. 4. Schematic diagram of the twist sensing setup.

Before we performed the twist experiment, the influence of the input light polarization state (controlled by the DPC 5500) on the transmission spectral response of the partially silver coated sample (40 s coating) was investigated and the result is shown in Fig. 5 (a). As one can see from the figure, the spectral dip strength is highly dependent on the polarization state of the input light. This effect could be understood as follows. Light polarization could be described as the vector sum of $\mathrm{S}$-polarized and P-polarized light components. For an uncoated HCF based fiber structure, the reflection coefficients $r_{1}$ and $r_{2}$ inside the HCF for non-normal incidence are given by the Fresnel equations (assuming air has a RI of 1 ):

$r_{1 \mathrm{~s}}=\frac{\cos \theta_{1}-n \cos \theta_{2}}{\cos \theta_{1}+n \cos \theta_{2}}, r_{2 \mathrm{~s}}=-r_{1 \mathrm{~s}}$
$r_{1 \mathrm{p}}=\frac{n \cos \theta_{1}-\cos \theta_{2}}{n \cos \theta_{1}+\cos \theta_{2}}, r_{2 \mathrm{p}}=-r_{1 \mathrm{p}}$

where $n$ is the RI of the cladding, $\theta_{1}$ and $\theta_{2}$ are the incident and refracted angles at the interface between the air core and cladding. According to equations (5) and (6), the reflection coefficient is dependent on the RI of the medium and on the 
angle of incidence. At normal incidence, light polarization state has no influence on the reflection coefficient. When the angle of incidence is different from normal and less than critical angle, the S-polarized light is always more strongly reflected than P-polarized light [28]. When the input linearly polarized (LP) light polarization state changes, its components in $\mathrm{P}$ and $\mathrm{S}$ directions change accordingly resulting in a variation of the reflection coefficients. For an ideal uncoated HCF, due to its circular symmetry, the polarization state of the input light has no influence on the reflection coefficient. However, a partial coating of silver on the outer surface of the HCF cladding significantly increases the polarization dependence of the reflection coefficient at the outer HCF surface because the one-side coating introduces an asymmetric RI distribution over the outer surface of the HCF (Fig. 5 (b)), leading to a large variation on the reflection coefficient of $r_{2}$, and consequently to large power variations as illustrated in Fig. 2. Besides, due to the actual uneven coating in all the directions, anisotropic properties and thus introduced birefringence could also introduce the change of the reflection coefficients at the cladding/silver interface. Let's assume now that a twist is applied to the partially silver coated HCF sensor. When LP light is launched into a circularly symmetric fibre, the light polarization state is maintained with almost no changes throughout the entire length of all fiber sections [29], but, due to the uneven silver coating on the HCF surface and hence the asymmetric RI distribution over the outer surface of the HCF, the $\mathrm{P}$ and $\mathrm{S}$ polarization components and their corresponding reflection coefficients of $r_{2}$ change due to the twist as shown in Fig. 5(c). Hence, the spectral dip strength varies with different twist angles. The experimental demonstration is shown below.

The effect of twist on the HCF sensor without the silver coating (T1) was firstly investigated and the experimental results are presented in Fig. 6. Before applying the twist, the polarization state of the input light was adjusted using the PC to achieve the largest dip strength. This initial spectrum with the largest dip strength was then labelled as a $0^{\circ}$ twist angle state. In our experiment, we define a clockwise twist as a positive twist angle while the counter clockwise twist as the negative twist angle. Fig. 6 shows the measured spectral dip strength variations under different twist angles. The maximum dip strength change is $2.18 \mathrm{~dB}$ in the case of twisting the HCF section (rotator 2), which is over 4 times higher than that of $0.51 \mathrm{~dB}$ for the case of twisting the SMF (rotator 1 ). This result indicates that twisting the HCF section achieves a higher sensitivity than that for the SMF alone. Ideally for a circularly symmetric fiber geometry, no spectral dip strength changes with twist would be expected, but this could change due to residual stress and core ellipticity, resulting from the practical fiber fabrication process. Especially for the HCF, any twistinduced stress could easily result in the RI change at the interface of inner air/cladding. Besides, the actual slight differences between the applied differential twists per unit length for section 1 and section 2 could also contribute to the observed difference in the twist sensitivities. It is noted that the fiber section containing HCF was fixed at $0^{\circ}$ twist angle while twisting of the SMF was performed, and vice versa.

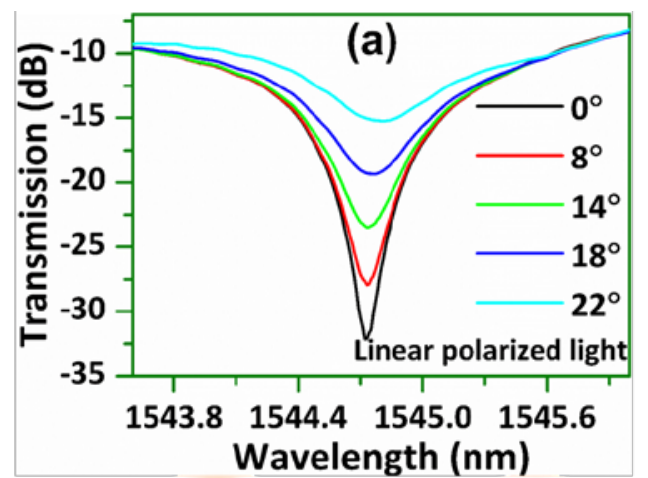

(b)
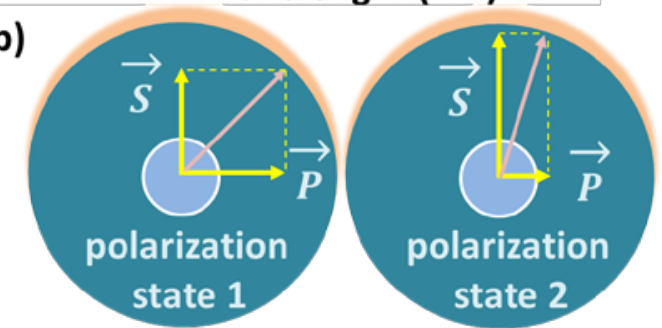

(c)
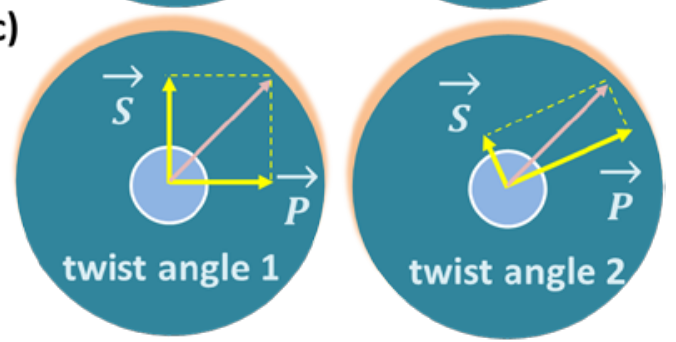

Fig. 5. Measured transmission spectral responses of the partially silver coated sample (40 s coating) for different input polarization states (angle of linearly polarized light changes from $0^{\circ}$ to $22^{\circ}$ ), and a schematic diagram shows the E-field orientation (b) at different LP state, and (c) at different twist angles.

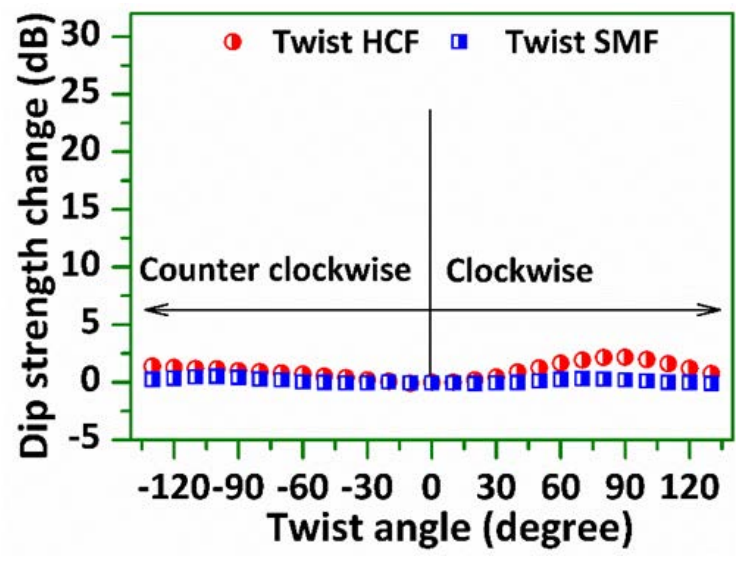

Fig. 6 Measured spectral dip strength variations for a range of twist angles by twisting SMF only and HCF only - uncoated HCF sensor (T1).

Next, the effect of twist on a partially silver coated HCF sensor was investigated. Samples T3 and T4 were selected for this experiment due to their relatively large dip strength.

Fig. 7 shows the corresponding experimental results for samples T3 and T4. An example of changes in the transmission dip strength for T3 at different twist angles is shown in Fig. 7(a). At $0^{\circ}$ of twist angle, the spectral dip has a high quality factor (Q) of $1.8 \times 10^{4}$ and a large extinction ratio (over $26 \mathrm{~dB}$ ). As the twist angle (in both clockwise and counter-clockwise directions) increases, the transmission dip strength decreases 
monotonically. Fig. 7(b) summarizes the changes of the spectral dip strength as the twist angle varies from $-90^{\circ}$ to $90^{\circ}$. It can be seen from the figure that the sensor has much higher sensitivity to twist in the small twist angles range. Comparing with the uncoated HCF sensor (T1), it was found that T3 has significantly improved sensitivities both when twisting the HCF and when twisting the SMF sections alone. The maximum sensitivity when twisting the SMF is $0.21 \mathrm{~dB} /{ }^{\circ}$ which is over 10 times higher than that of the uncoated HCF sensor. When the HCF section was twisted, a maximum sensitivity of $0.647 \mathrm{~dB} /{ }^{\circ}$ was achieved, which is over 17 times higher than that of the HCF sensor without the silver coating.
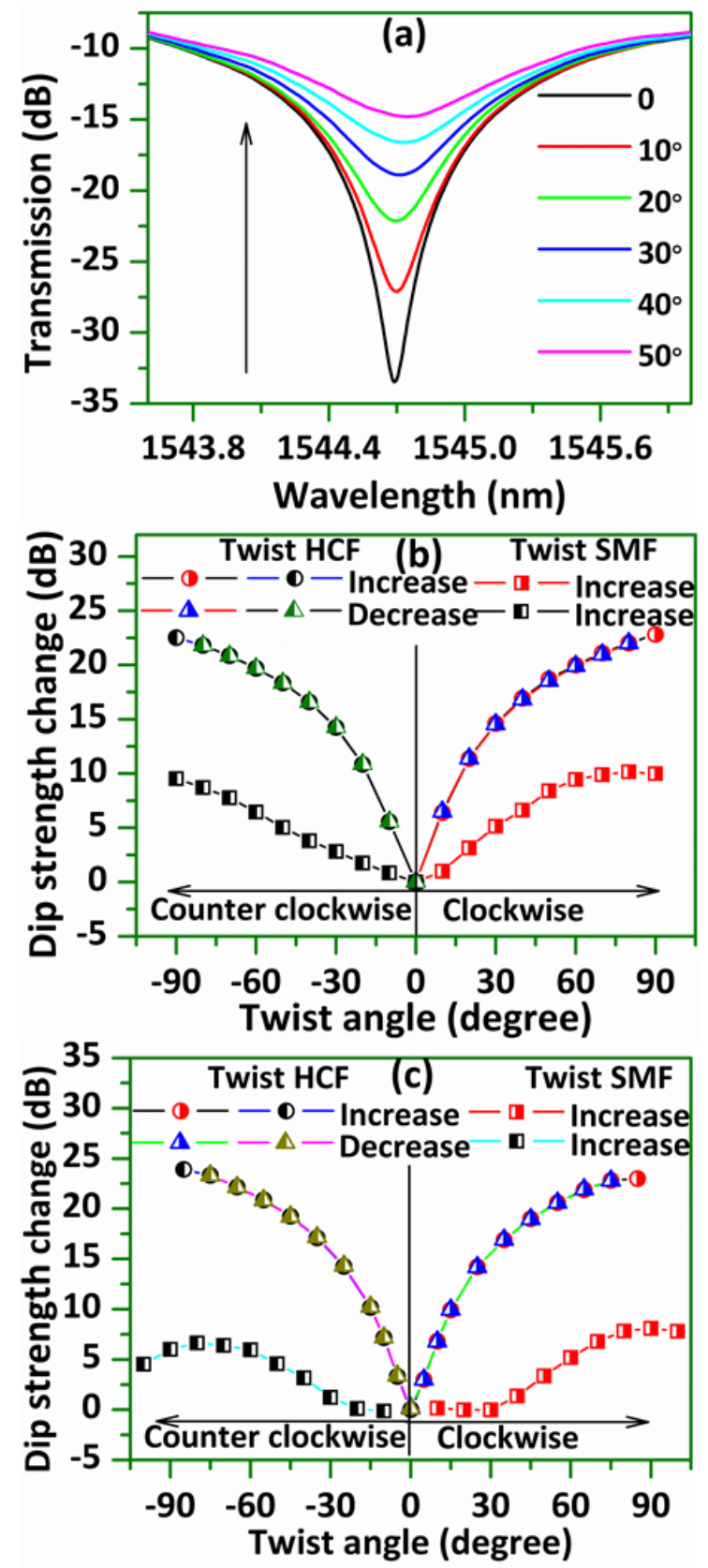

FIG. 7. (a) An example of the measured spectral response of T3 when twisting HCF; (b) measured dip strength change of T3 and (c) T4 for a range of twist angles when twisting SMF only and HCF only - partially silver coated HCF sensor.

The above experimental results can be explained as follows: since the reflection coefficient at the outer HCF surface after partial silver coating is polarization dependent, twisting of HCF will introduce significant change in the relative amplitude of the transmitted light in the air core due to the changed reflection coefficient, which results in the variations of the output light intensity as shown in Fig. 2 and Fig. 5. In addition, the sensor also demonstrates good repeatability of its response with no evidence of hysteresis since the measured data for the twist angle increasing cycle matches very well the data for the twist decreasing cycle. The twist performance of T4 was also tested and the results are plotted in Fig. 7(c), which shows similar to the T3 spectral changes but with slightly improved twist sensitivity. The maximum twist sensitivity is up to $0.717 \mathrm{~dB} /{ }^{\circ}$ in the range of twist angles from $0^{\circ}$ to $10^{\circ}$.

As can be seen from Fig. 7 (b), the dip strength decreases when the fiber is twisted in both clockwise and counter clockwise directions. Hence, a first order derivative of the dip strength change is employed to identify the twist direction as shown in Fig. 8(a), a positive derivative corresponds to a clockwise twist while a negative derivative indicates a counter clockwise twist. By using both dip strength variation and the first order derivative data, both the twist angle and twist direction can be clearly identified.
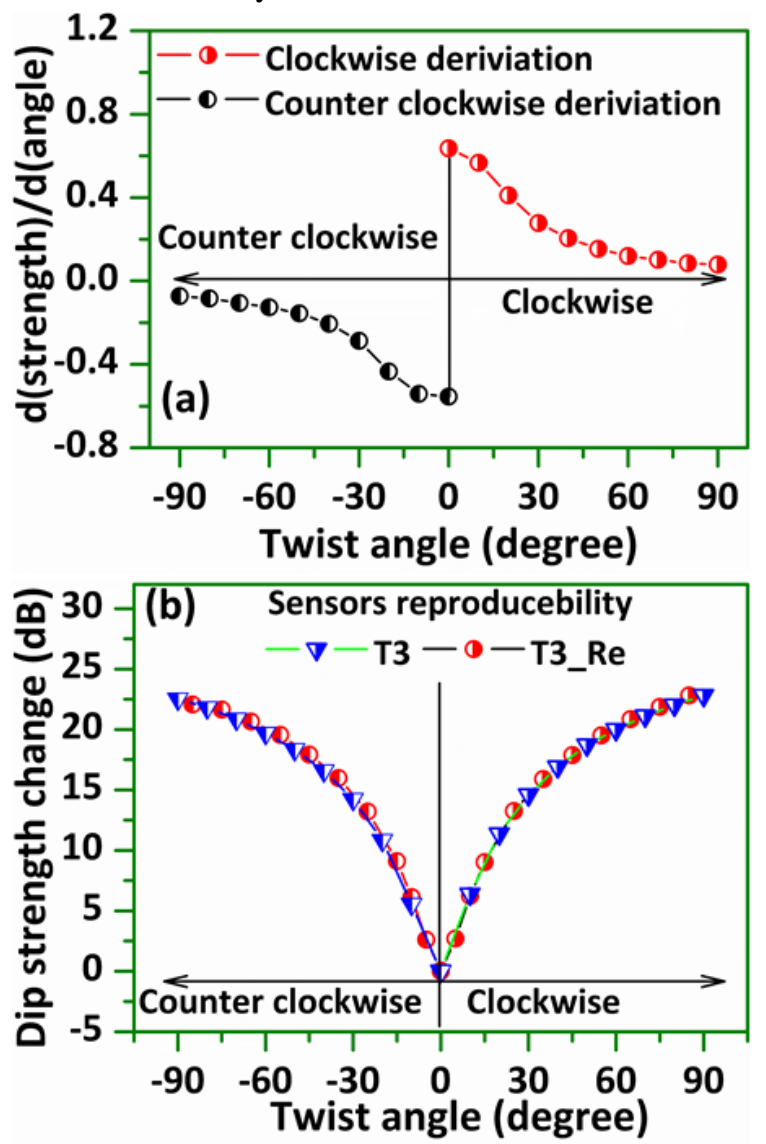

FIG. 8. (a) First order derivative of the measured spectral dip strength change with the twist angle when twisting HCF and (b) sensor's reproducibility demonstration with different samples when twisting the HCF.

It is noted that since the operating principle of our sensor is based on the spectral dip strength modulation, the reproducibility of the sensor is mainly dependent on the coating thickness and the bare HCF structure's spectral dip strength. In 
reality, there would always be some variations in the twist sensitivities for different sensor samples due to the variation in coating thickness and dip strength. It is also noted that the higher the sensitivity of the fiber structure, the less reproducible it is in practice. In our experiment, by choosing two bare HCF structure samples with similar spectral dip strengths and coating them with silver for the same time period of $40 \mathrm{~s}$, two sensor samples with very similar responses to twist were successfully prepared as shown in Fig. 8(b).

\section{CONCLUSION}

In summary, a novel high sensitivity twist sensor is proposed and investigated by partially coating a thin layer of silver film on the surface of a short section of HCF. Samples with different coating thicknesses have been studied. The experimental results demonstrate that the samples with $4.5 \mathrm{~nm}$ (40 s coating) and $6.7 \mathrm{~nm}$ (60 s coating) silver coating thickness have the highest sensitivity of $0.647 \mathrm{~dB} /{ }^{\circ}$ and $0.717 \mathrm{~dB} /{ }^{\circ}$ in the range of twist angles from $0^{\circ}$ to $10^{\circ}$, respectively. Moreover, twist of the HCF section has higher sensitivity than that of the SMF section for sensors both with and without the silver coating. Experiments proved that the proposed sensor also shows good repeatability of twist response.

\section{REFERENCES}

[1] G.W. Housner, L.A. Bergman, T.K. Caughey, A.G. Chassiakos, R.O. Claus, S.F. Masri, et al, "Structural control: past, present, and future," J. Eng. Mech., vol. 123, pp. 897-971, 1997.

[2] H.N. Li, D.S. Li, G.B. Song, "Recent applications of fiber optic sensors to health monitoring in civil engineering," Eng. Struct., vol. 26, pp. 1647-1657, 2004.

[3] Y. P. Wang and Y. J. Rao, "Long period fibre grating torsion sensor measuring twist rate and determining twist direction simultaneously," Electron. Lett., vol. 40, pp. 164-166, 2004.

[4] Y. J. Rao, T. Zhu, and Q. J. Mo, "Highly sensitive fiber-optic torsion sensor based on an ultra-long-period fiber grating," Opt. Commun. vol.266, pp. 187-190, 2006.

[5] Y. W., M. Wang, and X. Q. Huang, "In fiber Bragg grating twist sensor based on analysis of polarization dependent loss," Opt. Express, vol. 21, pp. 11913-11920, 2013.

[6] J. H. Wo, M. Jiang, M. Malnou, Q. Sun, J. Zhang, P. P. Shum, and D. Liu, "Twist sensor based on axial strain insensitive distributed Bragg reflector fiber laser,” Opt. Express, vol. 20, pp. 2844-2850, 2012.

[7] L. L. Shi, T. Zhu, Y. E. Fan, K. S. Chiang, and Y. J. Rao, "Torsion sensing with a fiber ring laser incorporating a pair of rotary long-period fiber gratings,” Opt. Commun. vol. 284, pp. 5299-5302, 2011.

[8] X. Chen, K. Zhou, L. Zhang, and I. Bennion, "In-fiber twist sensor based on a fiber Bragg grating with 81 tilted structure,” IEEE Photon. Technol. Lett., vol. 18, pp. 2596-2598, 2006.

[9] R. M. Silva, M. S. Ferreira, and O. Frazão, "Temperature independent torsion sensor using a high-birefringent Sagnac loop interferometer,” Opt. Commun., vol. 285, pp. 1167-1170, 2012.

[10] L. Dejan and D. Denis, "In-line, fiber-optic polarimetric twist/torsion sensor,” Opt. Lett., vol. 38, pp. 1494-1496, 2013.

[11] B. Huang, X. Shu, and Y. Du, "Intensity modulated torsion sensor based on optical fiber reflective Lyot filter," Opt. Express, vol. 25, pp. 5081-5090, 2017.

[12] T. Guo, F. Liu, F. Du, Z. Zhang, C. Li, B. O. Guan, and J. Albert, "VCSEL-powered and polarization-maintaining fiber-optic grating vector rotation sensor," Opt. Express, vol. 21, pp. 19097-19102, 2013.

[13] H. M. Kim, T. H. Kim, B. K. Kim, and Y. J. Chung, "Temperature-insensitive torsion sensor with enhanced sensitivity by use of a highly birefringent photonic crystal fiber," IEEE Photon. Technol. Lett., vol. 22, pp. 1539-1541, 2010.

[14] T. Hu, Y. Zhao, D. Wu, "Novel torsion sensor using a polarization maintaining photonic crystal fiber loop mirror,” Instrum. Sci. Technol. vol. 44, pp. 46-53, 2016.

[15] P. Zu, C. C. Chan, Y. X. Jin, T. X. Gong, Y. F. Zhang, L. H. Chen, and X. Y. Dong, "A temperature-insensitive twist sensor by using low-birefringence photonic-crystal-fiber-based Sagnac interferometer," IEEE Photon.Technol. Lett., vol. 23, pp. 920-922, 2011.

[16] O. Frazao, C. Jesus, J. M. Baptista, J. L. Santos, and P. Roy, "Fiber-optic interferometric torsion sensor based on a two-LP-mode operation in birefringent fiber,” IEEE Photon. Technol. Lett., vol. 21, pp. 1277-1279, 2009.

[17] W. Chen, S. Lou, L. Wang, H. Zou, W. Lu, and S. Jian, "Highly sensitive torsion sensor based on Sagnac interferometer using side-leakage photonic crystal fiber,” IEEE Photonics Technol. Lett., vol. 23, pp. 16391641, 2011.

[18] B. B. Song, Y. P. Miao, W. Lin, H. Zhang, J. X. Wu, and B. Liu, "Multi-mode interferometer-based twist sensor with low temperature sensitivity employing square coreless fibers," Opt. Express, vol. 21, pp. 26806-26811, 2013.

[19] D. Yu, Q. Mo, Z. Hong, S. Fu, C. Sima, M. Tang, and D. Liu, "Temperature-insensitive fiber twist sensor based on elliptical-core few-mode fiber,” Opt. Lett., vol. 41, pp. 4617-4620, 2016.

[20] O. Frazão, R. M. Silva, J. Kobelke, and K. Schuster, "Temperature- and strain-independent torsion sensor using a fiber loop mirror based on suspended twin-core fiber," Opt. Lett., vol. 35, pp. 2777-2779, 2010.

[21] C. L. Lee, L. H. Lee, H. E. Hwang, and J. M. Hsu, "Highly Sensitive Air-Gap Fiber Fabry-Pérot Interferometers Based on Polymer-Filled Hollow Core Fibers,” IEEE Photon. Tech. Lett., vol. 24, pp. 149-151, 2012.

[22] C. L. Lee, H. Y. Ho, J. H. Gu, T. Y. Yeh, and C. H. Tseng, "Dual hollow core fiber-based Fabry-Perot interferometer for measuring the thermo-optic coefficients of liquids," Opt. Lett., vol. 40, pp. 459-462, 2015.

[23] M. S. Ferreira, L. Coelho, K. Schuster, J. Kobelke, J. L. Santos, and O. Frazao, "Fabry-Perot cavity based on a diaphragm-free hollow-core silica tube,” Opt. Lett., vol. 36, pp. 4029-4031, 2011.

[24] D. Liu, Q. Wu, C. Mei, J. Yuan, et.al. "Hollow Core Fiber Based Interferometer for High Temperature $\left(1000{ }^{\circ} \mathrm{C}\right)$ Measurement”, J. Lightwave Techno., vol. 36, pp. 1583-1590, 2018.

[25] R. Gao, D. Lu, J. Cheng, Y. Jiang, L. Jiang, and Z. Qi, “Humidity sensor based on power leakage at resonance wavelengths of a hollow core fiber coated with reduced graphene oxide,” Sens. Actuat. Biol. Chem., vol. 222, pp. 618-624, 2016.

[26] R. Gao, Y. Jiang, and Y. Zhao, "Magnetic field sensor based on anti-resonant reflecting guidance in the magnetic gel-coated hollow core fiber,” Opt. Lett., vol. 39, pp. 6293-6296, 2014.

[27] A. M. Zheltikov, "Ray-optic analysis of the (bio)sensing ability of ring-cladding hollow waveguides,” Appl. Opt., vol. 47, pp. 474-479, 2008.

[28] C. A. Dimarzio, Optics for engineers. Boca Raton, USA, Crc Press, 2011, pp. 147.

[29] A.M. Smith, "Birefringence induced by bends and twists in single-mode optical fiber,” Appl. Opt., vol. 19, pp. 2606-2611, 1980. 